\title{
Changes in bone metabolism after gastric cancer surgery in male patients: a prospective observational study
}

\author{
Yosuke Atsumi ${ }^{1,2} \circledast$ - Yasushi Rino ${ }^{1} \cdot$ Hiroo Wada $^{2} \cdot$ Yuichi Kitani $^{2} \cdot$ Yukihiro Ozawa $^{2} \cdot$ Toru Aoyama $^{1} \cdot$ Takashi Oshima $^{1}$. \\ Norio Yukawa' ${ }^{1}$ Takaki Yoshikawa ${ }^{1,3} \cdot$ Munetaka Masuda $^{1}$
}

Received: 4 March 2018 / Accepted: 4 May 2018 / Published online: 10 May 2018

(c) The International Gastric Cancer Association and The Japanese Gastric Cancer Association 2018

\begin{abstract}
Background Several retrospective studies have shown that bone disorders occur after gastric cancer surgery. This study was designed to prospectively evaluate the changes in bone metabolism after gastrectomy for gastric cancer.

Methods We prospectively enrolled 39 men with early gastric cancer who underwent gastrectomy. We excluded women to avoid the effects of menopause. We employed dual energy X-ray absorptiometry (DEXA) to measure bone mineral density (BMD) of the lumbar spine. DEXA was performed before and 1 and 2 years after surgery. The serum levels of alkaline phosphatase (ALP), 1,25-dihydroxy vitamin D [1,25(OH $\left.)_{2} \mathrm{VD}\right]$, 25-hydroxy vitamin D [25(OH)VD], and estradiol were measured before surgery and every 3 months until 2 years after surgery.

Results DEXA revealed that BMD significantly decreased by $0.036 \pm 0.033 \mathrm{~g} / \mathrm{cm}^{2} 12$ months after gastrectomy $(P<0.001)$ and by $0.046 \pm 0.040 \mathrm{~g} / \mathrm{cm}^{2} 24$ months after gastrectomy $(P<0.001)$. The serum ALP level significantly increased by $38.31 \pm 103.8 \mathrm{IU} / \mathrm{L} 24$ months after surgery $(P=0.013)$. The serum $25(\mathrm{OH}) \mathrm{VD}$ level significantly decreased by $4.88 \pm 6.42 \mathrm{ng} /$ $\mathrm{ml} 24$ months after surgery $(P<0.001)$, whereas the serum $1,25(\mathrm{OH})_{2} \mathrm{VD}$ levels were consistently in the normal range. The serum estradiol level significantly increased by $2.94 \pm 7.49 \mathrm{pg} / \mathrm{ml} 12$ months after gastrectomy $(P=0.035)$. A lower preoperative body mass index (BMI) significantly correlated with the reduction in BMD 12 months after surgery; the correlation coefficient was $0.37(P=0.025)$.

Conclusions This study showed that a significant decrease in BMD was observed for up to 24 months after gastrectomy, not only 12 months.
\end{abstract}

Keywords Gastrectomy $\cdot$ Bone mineral density $\cdot T$ score $\cdot$ Osteoporosis

\section{Introduction}

In 2015, gastric cancer developed in about 1,313,000 people worldwide. Gastric cancer is the third most frequent cause of cancer-related death, coming after lung cancer and colorectal cancer [1]. Correct diagnosis and curative surgery have improved survival in patients with gastric cancer, and the

Yosuke Atsumi

yosuke31004@gmail.com

1 Department of Surgery, Yokohama City University, 3-9 Fukuura, Kanazawa-ku, Yokohama, Japan

2 Department of Surgery, Miura City Hospital, 4-33 Koyo-cho, Miura, Japan

3 Department of Gastrointestinal Surgery, Kanagawa Cancer Center, 2-3-2 Nakao, Asahi-ku, Yokohama, Japan
5 -year survival rate of patients with early gastric cancer is higher than $90 \%$ in Japan [2].

Several studies have reported that bone disorders occur after gastric cancer surgery [3-9]. Previous studies suggested that reduced secretion of gastric acid, loss of the digestive function of the stomach, and rapid transit through the small intestine can cause calcium and vitamin D deficiency [10], leading to hyperparathyroidism, bone mineral loss, bone disorders, and fractures [11]. Bone fractures associated with low bone mineral density (BMD) not only lower a patient's mobility capacity and quality of life, but also increase mortality. Previous meta-analyses have shown that lower BMD is associated with significantly increased risks of all-cause and cardiovascular mortality [12].

However, most previous studies of bone disorders after gastric cancer surgery were retrospective and had no data on BMD before surgery [3-9]. Therefore, when BMD begins 
to change and the extent of such change remain unknown. Bone metabolism in women differs from that in men because of differences in endocrinological factors, physical activity, and diet. Furthermore, the previous study demonstrated anticancer agents influenced bone metabolism [13]. The only previous prospective study that reported on changes in BMD after gastric cancer surgery enrolled not only men, but also women, and some patients received adjuvant chemotherapy. These factors might have, therefore, affected the results [14].

Consequently, our study aimed to prospectively assess changes in bone metabolism before and after early gastric cancer surgery in a group of only men.

\section{Methods}

This study was a prospective, single-center, observational study. A total of 42 consecutive male patients who underwent gastrectomy for pathologically Stage I cancer from April 2009 to March 2015 were enrolled. We excluded patients with severe kidney disease requiring dialysis, patients with severe liver disease, patients receiving treatment with bisphosphonates or any other bone-active drugs, including vitamin $\mathrm{D}$, and patients who were followed up for less than 2 years. After excluding patients who met the exclusion criteria, 39 men were evaluated.

This study was approved by the Institutional Review Board of Yokohama City University (Yokohama, Japan) and was conducted according to the principles of the Declaration of Helsinki. Informed consent was obtained from all patients.

We employed dual energy X-ray absorptiometry (DEXA) to measure BMD. The primary outcomes were BMD and $T$ score as measured by DEXA. The T-score is the standard deviation from the mean BMD of young normal adults, and the World Health Organization (WHO) defines "low bone mass" as bone density that is more than one standard deviation below the mean BMD in healthy young adults ( $T$ score $\leq-1)$, and "osteoporosis" as bone density that is $\geq 2.5$ standard deviations below this value $(T$ score $\leq-2.5$ ) [15]. The National Osteoporosis Foundation (NOF) recommends that pharmacologic treatment should be considered in patients with a $\mathrm{T}$-score of $\leq-2.5$, and in postmenopausal women and men older than 50 years with a $T$ score between $\leq-1.0$ and $>-2.5$ who have a high fracture risk [16]. The measurement site was the lumbar vertebrae (L2-L4). DEXA was performed before and 1 and 2 years after surgery. The serum levels of 1,25-dihydroxy vitamin $\mathrm{D}\left(1,25(\mathrm{OH})_{2} \mathrm{VD}\right)$, 25-hydroxy vitamin D (25(OH)VD), and alkaline phosphatase (ALP) were measured before surgery and every 3 months until 2 years after surgery. We also measured the serum estradiol (E2) level because we previously found that the serum E2 level increased in patients with progressive bone disorders after gastric cancer surgery [17]. The clinicopathological and perioperative data of all patients, including the calorie intake at discharge, were also assessed.

We used the Wilcoxon signed-rank test to compare preoperative and postoperative continuous variables (such as BMD), and Spearman`s correlation coefficient to determine the relations between continuous variables. The Kruskal-Wallis test was used to compare continuous variables among three or more groups. $P<0.05$ was considered to indicate statistical significance, and $P$ values between $<0.10$ and $\geq 0.05$ were considered to indicate marginal significance. All statistical analyses were performed with EZR (Saitama Medical Center, Jichi Medical University, Saitama, Japan), which is a graphic user interface for R (The R Foundation for Statistical Computing, Vienna, Australia). More precisely, it is a modified version of $\mathrm{R}$ commander designed to add statistical functions frequently used in biostatistics [18].

\section{Results}

\section{Patient characteristics}

The patient characteristics are shown in Tables 1 and 2 . The mean age and preoperative body mass index (BMI) of the patients were $67.2 \pm 10.1$ years and $23.3 \pm 3.0 \mathrm{~kg} /$

Table 1 Clinicopathological features of 39 patients(1)

\begin{tabular}{lll}
\hline Factor & Number of patients $\%$ \\
\hline$n$ & 39 & \\
$\begin{array}{l}\text { Age (years) } \\
\text { Sex }\end{array}$ & $67.2(10.1)$ & \\
$\quad$ Male/female & $39 / 0$ & $100 / 0$ \\
BMI $\left(\mathrm{kg} / \mathrm{m}^{2}\right)$ & $23.3(3.0)$ & \\
ECOG PS & & $100 / 0 / 0 / 0 / 0$ \\
0/1/2/3/4 & $39 / 0 / 0 / 0 / 0$ & $61.5 / 28.2 / 7.7 / 2.6$ \\
$\begin{array}{l}\text { Type of surgery } \\
\text { DG/TG/PG/partial resection }\end{array}$ & $24 / 11 / 3 / 1$ & $59.0 / 30.8 / 7.7 / 2.6$ \\
Reconstruction & & \\
BI/RY/EG/none & $23 / 12 / 3 / 1$ & $41.0 / 59.0$ \\
Depth of invasion & & $94.9 / 5.1$ \\
pT1a/pT1b & $16 / 23$ & $0 / 100$ \\
Nodal involvement & & \\
pN0/pN1 & $37 / 2$ & \\
Tumor recurrence & & \\
Yes/no & $0 / 39$ & \\
\hline
\end{tabular}

Continuous variables are shown as the mean (SD)

$B M I$ body mass index, ECOG-PS the Eastern Cooperative Oncology Group Performance Status, $D G$ distal gastrectomy, $T G$ total gastrectomy, $P G$ proximal gastrectomy, $B I$ Billroth-I reconstruction, $R Y$ Roux-en-Y reconstruction, $E G$ esophagogastrostomy 
Table 2 Clinicopathological features of 39 patients(2)

\begin{tabular}{llr}
\hline Factor & Normal range of men & Mean (SD) \\
\hline Preoperative lumber spine BMD $\left(\mathrm{g} / \mathrm{cm}^{2}\right)$ & & $1.00(0.21)$ \\
Preoperative lumber spine $T$ score & & $-0.38(1.80)$ \\
Preoperative serum ALP $(\mathrm{IU} / \mathrm{l})$ & $79.0-224.0$ & $223.5(59.7)$ \\
Preoperative serum 1,25(OH) $)_{2} \mathrm{VD}(\mathrm{pg} / \mathrm{ml})$ & $20.0-60.0$ & $60.5(14.9)$ \\
Preoperative serum 25(OH) VD $(\mathrm{ng} / \mathrm{ml})$ & $10.0-55.0$ & $29.3(8.1)$ \\
Preoperative serum estradiol $(\mathrm{pg} / \mathrm{ml})$ & $20.0-60.0$ & $21.1(8.9)$ \\
The amount of calorie intake at discharge $(\mathrm{kcal})$ & & $1187.8(350.0)$ \\
\hline
\end{tabular}

$S D$ Standard deviation, $B M D$ bone mineral density, $A L P$ Alkaline Phosphatase, 1,25( $\mathrm{OH})_{2} V D$ 1,25 dihydroxyvitamin $\mathrm{D}, 25(\mathrm{OH}) V D 25$ hydroxyvitamin D $\mathrm{m}^{2}$, respectively. All patients had an Eastern Cooperative Oncology Group Performance Status (ECOG-PS) of 0 and a mucosal or submucosal depth of tumor invasion. Distal gastrectomy (DG), total gastrectomy (TG), proximal gastrectomy (PG), and partial gastric resection were performed in 24 patients, 11 patients, 3 patients, and 1 patient, respectively. Billroth-I (BI), Roux-en-Y (RY), and esophagogastrostomy (EG) reconstruction were performed in 23 patients, 12 patients, and 3 patients, respectively. The patients' body weights significantly decreased by $7.0 \pm 12.1 \mathrm{~kg} 12$ months after surgery and $7.9 \pm 11.5 \mathrm{~kg}$ 24 months after surgery $(P=0.002$ and $P<0.001$, respectively) (Fig. 1a). No patient had recurrence during follow-up.

\section{Changes in bone metabolism after surgery}

DEXA revealed that BMD and $T$ score had significantly decreased 12 months after surgery by $0.036 \pm 0.033 \mathrm{~g} /$ $\mathrm{cm}^{2}(P<0.001)$ and $0.31 \pm 0.28(P<0.001)$, respectively. In addition, both of these variables further decreased at 24 months (Fig. 1b, c). The percent changes from the baseline BMD were $-4.03 \% 12$ months and $-4.99 \% 24$ months after surgery. The number of patients with a $T$ score of $\leqq-1$ (low bone mass and osteoporosis) at 12 months was $21 / 39$ (53.9\%), and the number of patients with a $T$ score that changed from normal to $\leqq-1$ (low bone mass) at 12 months was 4/39 (10.3\%). The serum ALP level significantly increased by $40.0 \pm 58.4 \mathrm{IU} / 19$ months after surgery and by $38.3 \pm 103.8 \mathrm{IU} / \mathrm{l} 24$ months after surgery $(P<0.001$
Fig. 1 a Body weight before and after surgery. b Bone mineral density (BMD) before and after surgery. $\mathbf{c} T$ score before and after surgery

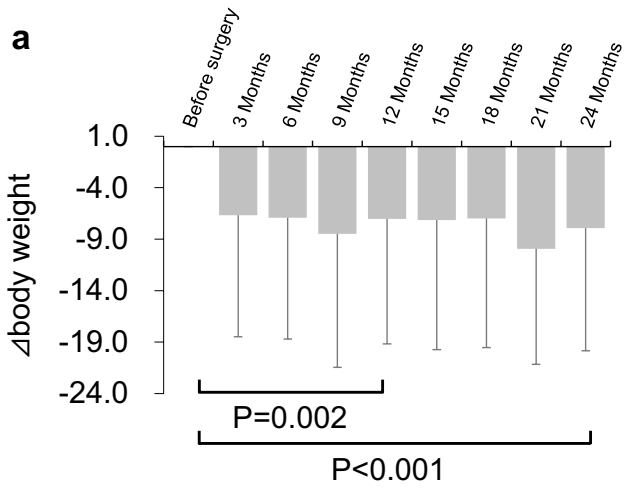

b

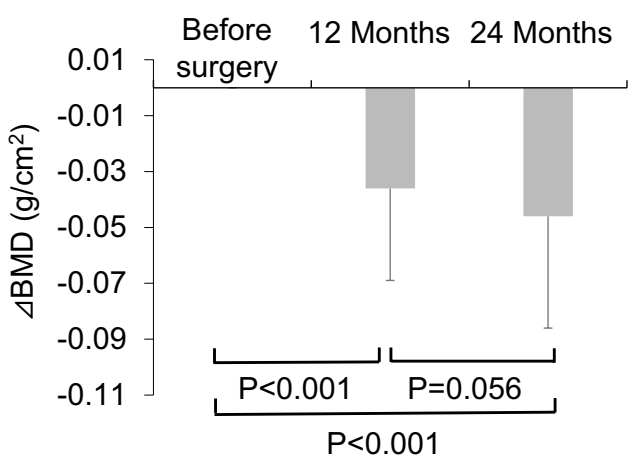

C

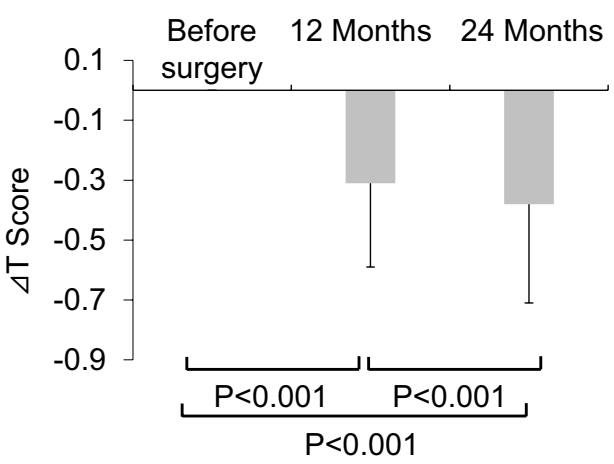


and $P=0.013$, respectively) (Fig. 2a). The serum $25(\mathrm{OH})$ VD level significantly decreased by $5.6 \pm 5.5 \mathrm{ng} / \mathrm{ml} 9$ months after surgery and by $4.9 \pm 6.4 \mathrm{ng} / \mathrm{ml} 24$ months after surgery $(P<0.001$ for both) (Fig. 2b). On the other hand, the serum $1,25(\mathrm{OH})_{2} \mathrm{VD}$ levels were consistently in the normal range (Fig. 2c). The serum E2 level significantly increased by $2.9 \pm 7.5 \mathrm{pg} / \mathrm{ml} 12$ months after gastrectomy $(P=0.035)$

(Fig. 2d).

\section{Relations between the rates of change in BMD and other factors in patients with gastric cancer}

We examined whether the rates of change in the serum ALP, 25(OH)VD, 1,25(OH)VD, and E2 levels were associated with the rates of change in BMD after gastrectomy. There was no significant correlation between the rates of change in the serum ALP, 25(OH)VD, 1,25(OH)VD and E2 levels, and the rates of change in the BMD 12 and 24 months after surgery. To assess predictors of a reduction in BMD, we examined the relations of the rates of change in BMD in patients with gastric cancer to body weight and the amount of calorie intake at discharge. There was no significant correlation between the rate of change in BMD and the change in body weight at 12 or 24 months after surgery or the amount of calorie intake at discharge, but there was a significant correlation between the change in BMD 12 months after surgery and the preoperative $\mathrm{BMI}$; the correlation coefficients was $0.37(P=0.025)$ (Fig. 3b, c).

\section{Discussion}

This study prospectively investigated the changes in bone metabolism after gastric cancer surgery in a group of only men. The results showed that BMD and $T$ score significantly decreased as early as 1 year after gastrectomy, and the proportion of patients with low bone mass and osteoporosis 1 year after gastrectomy was as high as $53.9 \%$. At first sight, the decrease of BMD shown in our study $(-0.036 \pm 0.033 \mathrm{~g} /$ $\mathrm{cm}^{2},-4.03 \%, T$ score $\left.-0.31 \pm 0.28\right)$ seemed a little change, however previous cross-sectional studies reported that the vertebral BMD in normal adult men decreased linearly by $0.0021 \mathrm{~g} / \mathrm{cm}^{2}$ per year and by $5 \%$ in 10 years $[19,20]$. Compared with those reports, bone loss in patients after gastrectomy in our study was considerably higher.

The previous prospective study demonstrated that bone loss occurred in the early postgastrectomy period consistent with the results of our study [14]. Our study assessed not only BMD but also $T$ scores and found that more than half of the patients had low bone mass or osteoporosis 1 year after gastrectomy; therefore, more than half the patients might require pharmacologic treatment within a year after gastrectomy [16]. Although we have reported the effectiveness of active vitamin D3 agents and alendronate for bone disorders after gastrectomy [21,22], pharmacologic treatment remains to be established. Our results will most likely facilitate discussions of when treatment for bone disorders should be begun in patients who undergo gastrectomy.
Fig. 2 a Serum alkaline phosphatase (ALP) levels before and after surgery. b Serum 25-hydroxy vitamin $\mathrm{D}[25(\mathrm{OH})$ VD] levels before and after surgery. c Serum 1,25-dihydroxy vitamin D [1,25(OH)2VD] levels before and after surgery. d Serum estradiol levels before and after surgery
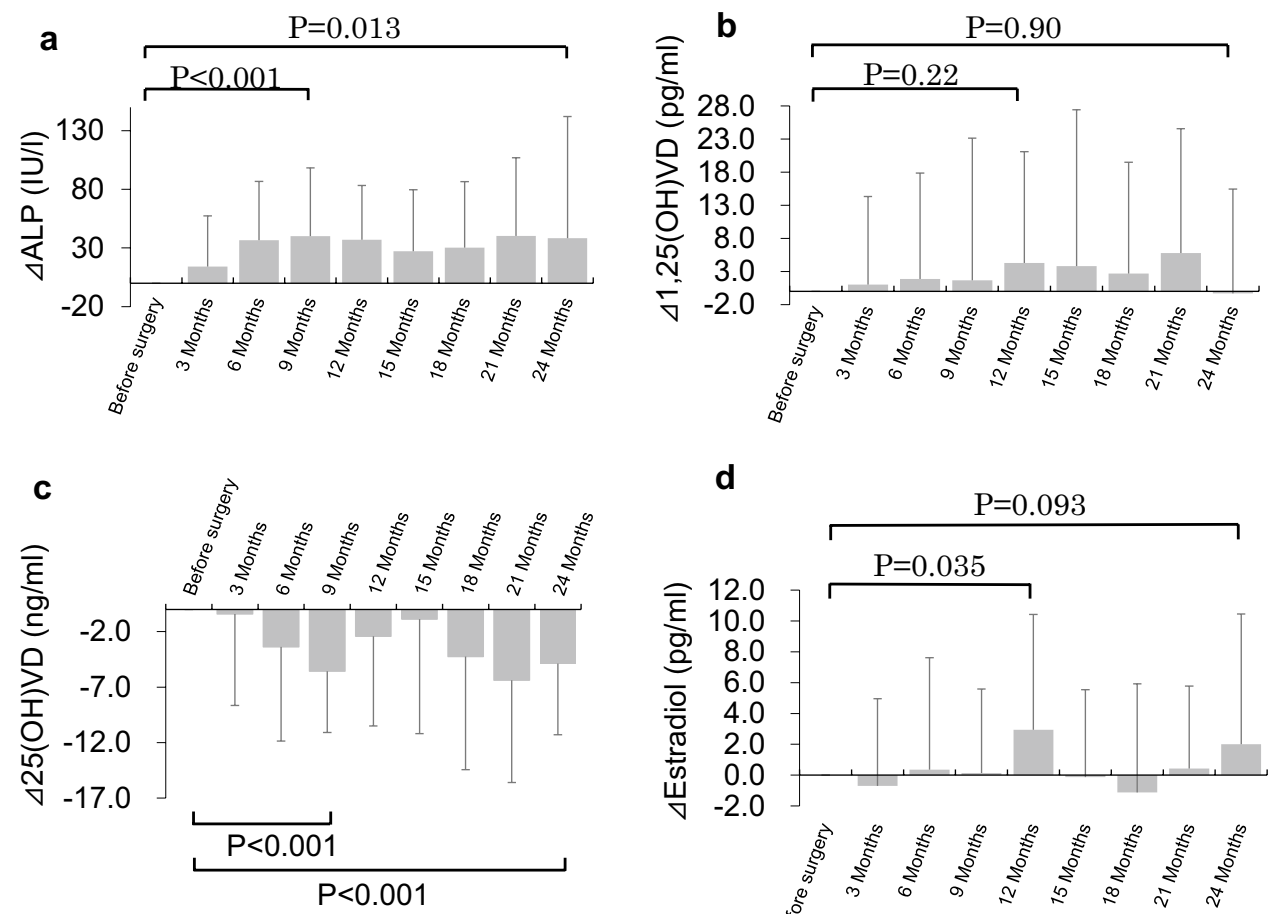

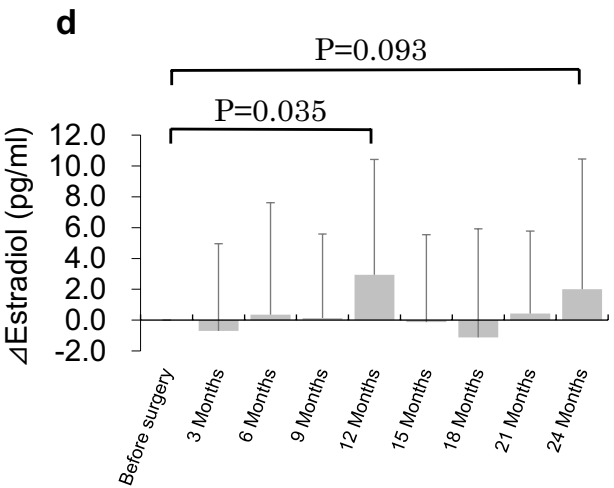


Fig. 3 a Relation between the change in the bone mineral density (BMD) and the change in the serum alkaline phosphatase (ALP) level 24 months after gastrectomy. b Relation between the change in the bone mineral density (BMD) 24 months after gastrectomy and the amount of calorie intake at discharge. c Relation between the bone mineral density (BMD) 12 months after gastrectomy and the preoperative body mass index (BMI)
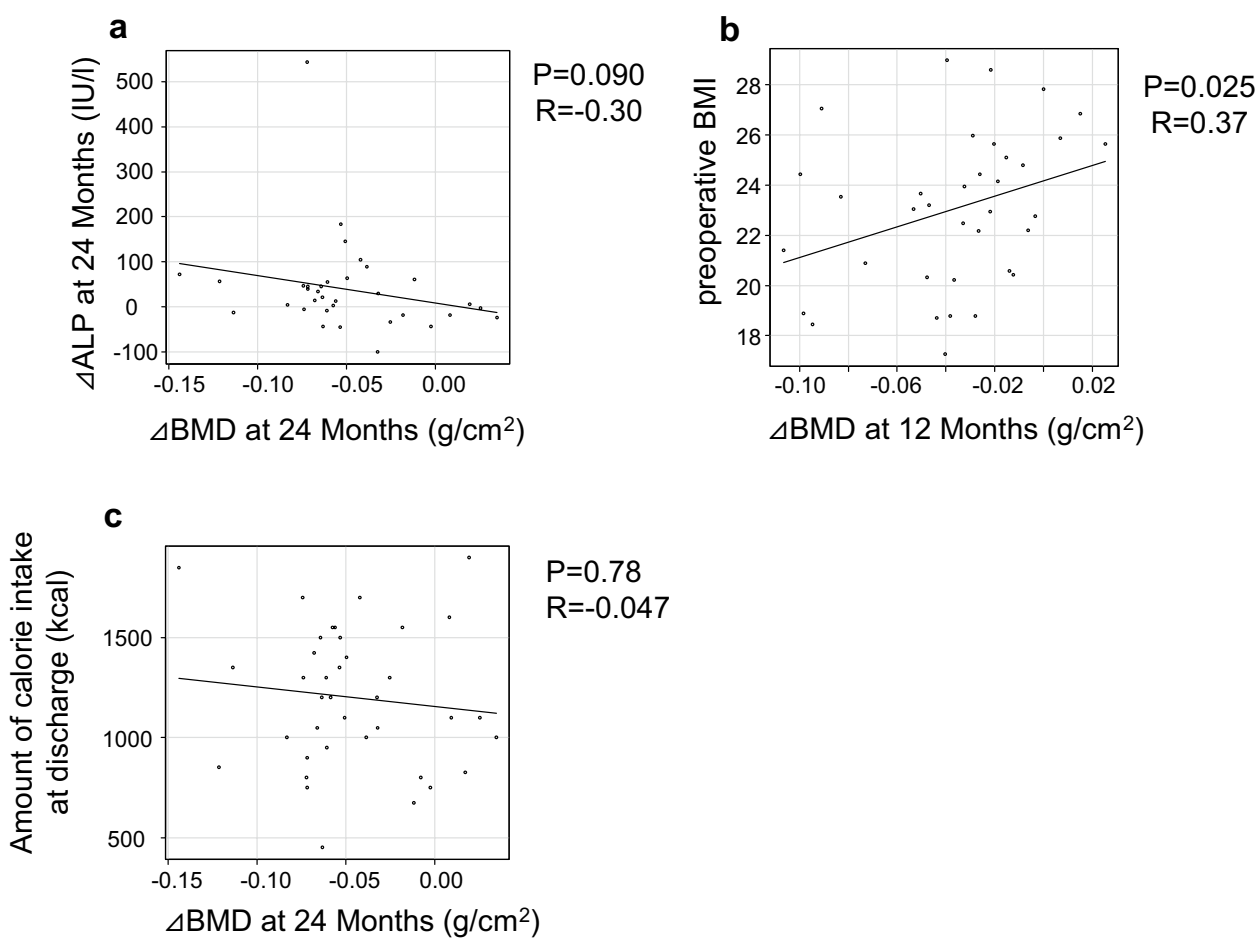

Furthermore, we demonstrated that low preoperative BMI was associated with the decrease in BMD 1 year after surgery. One of risk factors for primary osteoporotic fractures identified by the World Health Organization included low BMI [23]. A low preoperative BMI might be a predictor of a decrease in BMD and the risk of osteoporotic fractures after gastric cancer surgery.

We previously reported that reduced food consumption after gastrectomy can cause BMD to decrease [24]. The present study demonstrated that the percent changes from the baseline BMD were $-4.03 \%$ in the first postoperative year and $-0.96 \%$ in the second postoperative year. Previous studies reported that food consumption decreases in the first postoperative year and gradually improves subsequently [25-27]. Our study suggested that reduced food consumption in the early postgastrectomy period strongly affected the BMD, which decreased by $4 \%$ in year 1 after surgery. In year 2 , food consumption gradually improved and the rate of decrease in the BMD was reduced.

After gastrectomy, the loss of stomach function and rapid pass through the small intestine cause malabsorption of fatsoluble vitamins [28]. Vitamin D, which is a fat-soluble vitamin, is only not absorbed in the small intestine, but is also produced in the skin in response to sunlight. Vitamin D is hydroxylated to $25(\mathrm{OH}) \mathrm{VD}$ in the liver and hydroxylated to $1,25(\mathrm{OH})_{2} \mathrm{VD}$ in the kidney. $1,25(\mathrm{OH})_{2} \mathrm{VD}$ is the most active metabolite of vitamin $\mathrm{D}$ and plays a very important role in the regulation of calcium and bone metabolism. The present study prospectively demonstrated that the serum 25(OH)VD level significantly decreased after gastrectomy, whereas the serum $1,25(\mathrm{OH})_{2}$ VD level increased slightly [10]. These findings suggest that a homeostatic response maintained the serum level of the most active metabolite of vitamin D.

E2 is the most common type of estrogen hormone. E2 suppresses osteoclasts and prevents osteoporosis by directly acting on osteoblasts. Previous studies reported that reduced bone mass and fractures increased not only in females but also males with low E2 levels [29-31]. These reports suggested that E2 is the regulator of bone metabolism in men. The present study showed that the serum E2 level increased significantly after gastrectomy. This finding suggested that elevation of the serum E2 level was a feedback reaction to inhibit bone loss.

Our study had several important limitations. First, it had a small sample size and was performed in a single center. We performed only univariate analysis, and cannot rule out the possibility that our findings were observed by chance. Second, our study was short-term and assessed follow-up data obtained within 2 years. Bone metabolism 3 years after gastrectomy and subsequently thus remains unknown. A third limitation was that the serum calcium level was not estimated in this study. The relation between the change in BMD and the serum calcium level thus also remains unknown. We could not measure also bone metabolic markers such as bone-specific alkaline phosphatase (BAP), tartrate-resistant acid phosphatase (TRACP), type 1 
collagen cross-linked $N$-telopeptide (NTX), and type $1 \mathrm{col}$ lagen C-terminal telopeptide (CTX) due to cost limitations. Given these limitations, changes in BMD and the relations between bone disorders after gastric cancer surgery and clinicopathological and perioperative factors including serum calcium and bone metabolic markers should be investigated in a large, long-term prospective study.

\section{Conclusions}

Our analysis suggests that the BMD of patients with gastric cancer decreases in the early postgastrectomy period, and low preoperative BMI might be a high risk factor for bone disorders after gastric cancer surgery.

Funding This study was funded by the Ministry of Education, Culture, Sports, Science and Technology of Japan, which provided Grants-inaid for Scientific Research, the Japan Society for the Promotion of Science (19591553).

\section{Compliance with ethical standards}

Conflict of interest We are indebted to the following organizations for providing funding for this study: Daiichi Sankyo, Inc., Tsumura \& Co., Johnson and Johnson, Taiho Pharmaceutical Co., Ltd., Covidien Japan, Inc., Asahi Kasei Pharma Corporation, Kyowa Hakko Kirin Co. Ltd., and Yakult Honsha Co., Ltd..

Human rights statement All procedures followed were in accordance with the ethical standards of the responsible committee on human experimentation (institutional and national) and with the Helsinki Declaration of 1964 and later versions.

Informed consent Informed consent was obtained from all patients for being included in the study.

\section{References}

1. Fitzmaurice C, Allen C, Barber RM, Barregard L, Bhutta ZA, Brenner $\mathrm{H}$, et al. Global, regional, and national cancer incidence, mortality, years of life lost, years lived with disability, and disability-adjusted life-years for 32 cancer groups, 1990-2015: a systematic analysis for the global burden of disease study. JAMA Oncol. 2017;3:524-48.

2. Sano T, Sasako M, Kinoshita T, Maruyama K. Recurrence of early gastric cancer. Follow-up of 1475 patients and review of the Japanese literature. Cancer. 1993;72:3174-8.

3. Sarasin C. Osteomalacie and hypochrome aneaemie nach magenresektion. Gastroenterology. 1941;66:182-97.

4. Inoue K, Shiomi K, Higashide S, Kan N, Nio Y, Tobe T, et al. Metabolic bone disease following gastrectomy: assessment by dual energy X-ray absorptiometry. Br J Surg. 1992;79:321-4.

5. Kobayashi S, Takahashi C, Kuroda T, Sugenoya A, Iida F, Katoh $\mathrm{K}$. Calcium regulating hormones and bone mineral content in patients after subtotal gastrectomy. Surg Today. 1994;24:295-8.

6. Liedman B, Bosaeus I, Mellstrom D, Lundell L. Osteoporosis after total gastrectomy. Results of a prospective, clinical study. Scand J Gastroenterol. 1997;32:1090-5.
7. Mellstrom D, Johansson C, Johnell O, Lindstedt G, Lundberg PA, Obrant K, et al. Osteoporosis, metabolic aberrations, and increased risk for vertebral fractures after partial gastrectomy. Calcified Tissue Int. 1993;53:370-7.

8. Nishimura O, Furumoto T, Nosaka K, Kouno K, Sumikawa M, Hisaki $\mathrm{T}$, et al. Bone disorder following partial and total gastrectomy with reference to bone mineral content. Jpn J Surg. 1986;16:98-105.

9. Rino Y, Takanashi Y, Yamamoto Y, Inagaki D, Kawamoto M, Harada $\mathrm{H}$, et al. Bone disorder and vitamin $\mathrm{D}$ after gastric cancer surgery. Hepato-gastroenterology. 2007;54:1596-600.

10. Rino Y, Yamamoto Y, Wada N, Yukawa N, Murakami H, Tamagawa $\mathrm{H}$, et al. Changes in vitamin D after gastrectomy. Gastric Cancer. 2007;10:228-33.

11. Heaney RP. Health is better at serum $25(\mathrm{OH}) \mathrm{D}$ above $30 \mathrm{ng} / \mathrm{mL}$. J Steroid Biochem Mol Biol. 2013;136:224-8.

12. Qu X, Huang X, Jin F, Wang H, Hao Y, Tang T, et al. Bone mineral density and all-cause, cardiovascular and stroke mortality: a meta-analysis of prospective cohort studies. Int J Cardiol. 2013;166:385-93.

13. Nakamura M, Ishiguro A, Muranaka T, Fukushima H, Yuki S, Ono $\mathrm{K}$, et al. A prospective observational study on effect of short-term periodic steroid premedication on bone metabolism in gastrointestinal cancer (ESPRESSO-01). Oncologist. 2017;22:592-600.

14. Baek KH, Jeon HM, Lee SS, Lim DJ, Oh KW, Lee WY, et al. Short-term changes in bone and mineral metabolism following gastrectomy in gastric cancer patients. Bone. 2008;42:61-7.

15. Kanis JA, Melton LJ 3rd, Christiansen C, Johnston CC, Khaltaev N. The diagnosis of osteoporosis. J Bone Miner Res. 1994;9:1137-41.

16. Cosman F, de Beur SJ, LeBoff MS, Lewiecki EM, Tanner B, Randall S, et al. Clinician's guide to prevention and treatment of osteoporosis. Osteoporos Int. 2014;25:2359-81.

17. Rino Y, Yukawa N, Yamamoto N, Ohshima T, Sato T, Tamagawa $\mathrm{H}$, et al. Self-defensive response to bone disorder after gastric cancer surgery. Clin Exp Med Sci. 2014;2:1-10.

18. Kanda Y. Investigation of the freely available easy-to-use software 'EZR' for medical statistics. Bone Marrow Transpl. 2013;48:452-8.

19. Kin K, Kushida K, Yamazaki K, Okamoto S, Inoue T. Bone mineral density of the spine in normal Japanese subjects using dualenergy X-ray absorptiometry: effect of obesity and menopausal status. Calcified Tissue Int. 1991;49:101-6.

20. Riggs BL, Wahner HW, Dunn WL, Mazess RB, Offord KP, Melton LJ 3rd. Differential changes in bone mineral density of the appendicular and axial skeleton with aging: relationship to spinal osteoporosis. J Clin Investigation. 1981;67:328-35.

21. Atsumi Y, Rino Y, Sato T, Cho H, Yoshikawa T, Yamamoto N, et al. Effectiveness of alendronate for bone disorder after gastrectomy for gastric cancer. Asian J Surg Asian Surg Assoc. 2017;40:470-4.

22. Rino Y, Imada T, Yamamoto Y, Takahashi M, Amano T, Takanashi Y. The efficacy of 1 alpha hydroxy vitamin D3 treatment of the metabolic bone disorder in patients who underwent gastrectomy for gastric cancer. Hepato-gastroenterology. 2000;47:1498-500.

23. Kanis JA, on behalf of the WHO Scientific Group. Assessment of osteoporosis at the primary health care level. WHO Collaborating Centre for Metabolic Bone Diseases. In: WHO Collaborating Centre for Metabolic Bone Diseases, University of Sheffield. 2007.

24. Rino Y. Clinical study of the relationship between a metabolic bone disorder and an amount of daily food intake. Yokohama Med J. 1996;47:25-9.

25. Karanicolas PJ, Graham D, Gonen M, Strong VE, Brennan MF, Coit DG. Quality of life after gastrectomy for adenocarcinoma: a prospective cohort study. Ann Surg. 2013;257:1039-46. 
26. Saeki H, Rino Y, Cho H, Sato T, Kawamoto M, Takanashi Y, et al. Evaluation of calorie intake according to age and sex in patients undergoing surgery for gastric cancer. Hepato-gastroenterology. 2008;55:795-8.

27. Yu W, Park KB, Chung HY, Kwon OK, Lee SS. Chronological changes of quality of life in long-term survivors after gastrectomy for gastric cancer. Cancer Res Treatment. 2016;48:1030-6.

28. Rino Y, Oshima T, Yoshikawa T. Changes in fat-soluble vitamin levels after gastrectomy for gastric cancer. Surg Today. 2017;47:145-50.
29. Bilezikian JP, Morishima A, Bell J, Grumbach MM. Increased bone mass as a result of estrogen therapy in a man with aromatase deficiency. N Engl J Med. 1998;339:599-603.

30. Morishima A, Grumbach MM, Simpson ER, Fisher C, Qin K. Aromatase deficiency in male and female siblings caused by a novel mutation and the physiological role of estrogens. J Clin Endocrinol Metab. 1995;80:3689-98.

31. Vandenput L, Ohlsson C. Estrogens as regulators of bone health in men. Nat Rev Endocrinol. 2009;5:437-43. 